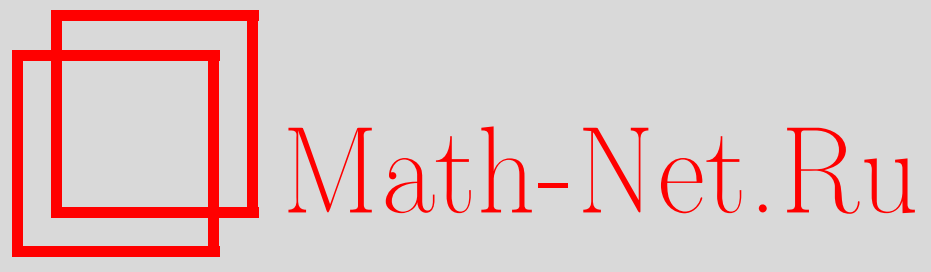

А. Г. Смирнов, О свойствах локализуемости высокосингулярных обобщенных функций, ТМФ, 2007, том 151, номер 2, 179-194

DOI: https://doi.org/10.4213/tmf6038

Использование Общероссийского математического портала Math-Net.Ru подразумевает, что вы прочитали и согласны с пользовательским соглашением http://www . mathnet.ru/rus/agreement

Параметры загрузки:

IP: 54.174 .149 .18

26 апреля 2023 г., 13:38:50

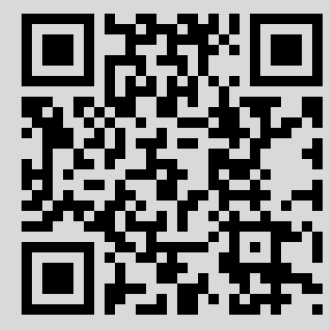




\title{
О СВОЙСТВАХ ЛОКАЛИЗУЕМОСТИ ВЫСОКОСИНГУЛЯРНЫХ ОБОБЩЕННЫХ ФУНКЦИЙ
}

\begin{abstract}
Исследованы свойства локализуемости обобщенных функций, определенных на широком классе пространств целых аналитических пробных функций. Использование данного класса, включающего все пространства Гельфанда-Шилова $S_{\alpha}^{\beta}\left(\mathbb{R}^{k}\right)$ с $\beta<1$, дает удобный язык для описания квантовых полей с высокосингулярным инфракрасным поведением. Показано, что для рассматриваемых аналитических функционалов может быть корректно определено понятие несущего конуса, заменяющее понятие носителя. В частности, доказано, что каждый функционал обладает однозначно определенным минимальным несущим конусом.
\end{abstract}

Ключевые слова: обобщенные функции, аналитические функционалы, инфракрасные сингулярности, несущие конусы, плюрисубгармонические функции, $L_{2}$-оценки Хермандера.

\section{1. ВВЕДЕНИЕ}

Данная статья посвящена изучению свойств локализуемости обобщенных функций, определенных на пространствах целых аналитических пробных функций. Обычное определение носителя обобщенной функции в этом случае неприменимо изза отсутствия пробных функций с компактным носителем (эта трудность хорошо известна в теории гиперфункций, где используются вещественно-аналитические пробные функции; см., например, главу 9 книги [1]). Проблема отыскания адекватной замены понятию носителя важна для распространения вайтмановского аксиоматического подхода на квантовые калибровочные теории. Из-за сильных инфракрасных сингулярностей калибровочные поля, вообще говоря, корректно определены лишь при усреднении с целыми аналитическими функциями в импульсном пространстве (это верно, например, для модели Швингера в произвольной $\alpha$-калибровке [2]) и, следовательно, не могут рассматриваться ни в рамках исходной вайтмановской формулировки [3], использующей распределения умеренного роста, ни в рамках более

\footnotetext{
*Физический институт им. П. Н. Лебедева РАН, Москва, Россия. E-mail: smirnov@lpi.ru
} 
общего подхода [4], основанного на фурье-гиперфункциях. В этой связи возникает проблема обобщения спектрального условия [5], стандартная формулировка которого в терминах вакуумных средних существенно использует понятие носителя обобщенной функции.

В работах [6], [7] исследовались свойства локализуемости функционалов, определенных на пространствах Гельфанда-Шилова $S_{\alpha}^{\beta}$ при $\beta<1$ (см. [8] по поводу определения и свойств пространств $S_{\alpha}^{\beta}$; если $\beta<1$, то $S_{\alpha}^{\beta}$ состоит из целых аналитических функций). Было показано, что для таких функционалов можно корректно ввести понятие несущего конуса, заменяющее понятие носителя. В частности, было доказано, что каждый элемент пространства $S_{\alpha}^{\prime \beta}\left(\mathbb{R}^{k}\right)$ (топологического сопряженного к $\left.S_{\alpha}^{\beta}\left(\mathbb{R}^{k}\right)\right)$ обладает однозначно определенным минимальным несущим конусом. В данной статье результаты работ [6], [7] распространяются на более широкий класс пространств пробных функций, использовавшийся ранее при анализе спектральных свойств сумм бесконечных рядов по виковым степеням свободных полей с индефинитной метрикой [9]. Данный класс определяется следующим образом.

ОПРЕДЕЛЕНИЕ 1. Пусть $\alpha(s)$ и $\beta(s)$ - непрерывные, монотонно неограниченно возрастающие функции на полуоси $s \geqslant 0$, причем функция $\beta$ выпукла и найдется такая константа $\varkappa>0$, что функция $\alpha(s) / s^{\varkappa}$ не убывает при достаточно больших $s$. Для любых $A, B>0$ обозначим через $\mathcal{E}_{\alpha, A}^{\beta, B}\left(\mathbb{R}^{k}\right)$ банахово пространство, состоящее из всех целых аналитических функций на $\mathbb{C}^{k}$ с конечной нормой

$$
\sup _{z=x+i y \in \mathbb{C}^{k}}|f(z)| e^{\alpha(|x / A|)-\beta(B|y|)} .
$$

Пространство $\mathcal{E}_{\alpha}^{\beta}\left(\mathbb{R}^{k}\right)$ определим как объединение $\bigcup_{A, B>0} \mathcal{E}_{\alpha, A}^{\beta, B}\left(\mathbb{R}^{k}\right)$, наделенное топологией индуктивного предела.

Для определенности норма $|\cdot|$ на $\mathbb{C}^{k}$ всюду будет предполагаться равномерной: $|z|=\max _{1 \leqslant j \leqslant k}\left|z_{j}\right|$. Если функция $\alpha$ выпукла, то пространства $\mathcal{E}_{\alpha}^{\beta}$ совпадают с пространствами типа $W$, описанными в главе 1 книги [10]. Если $\alpha(s)=s^{1 / \mu}$ и $\beta(s)=s^{1 /(\nu-1)}, \nu<1$, то $\mathcal{E}_{\alpha}^{\beta}\left(\mathbb{R}^{k}\right)=S_{\mu}^{\nu}\left(\mathbb{R}^{k}\right)$ (во избежание путаницы мы использовали обозначение $S_{\mu}^{\nu}$ вместо стандартного $S_{\alpha}^{\beta}$ ). Будем называть конус $W$ конической окрестностью конуса $U$, если $W$ имеет открытую проекцию ${ }^{1)}$ и содержит $U$. Определение несущих конусов основано на введении наряду с пространствами $\mathcal{E}_{\alpha}^{\beta}\left(\mathbb{R}^{k}\right)$ родственных пространств, ассоциированных с конусами в $\mathbb{R}^{k}$.

ОПРеДЕЛЕниЕ 2. Пусть $U$ - непустой конус в $\mathbb{R}^{k}$, а функции $\alpha$ и $\beta$ удовлетворяют условиям определения 1 . Для любых $A, B>0$ обозначим через $\mathcal{E}_{\alpha, A}^{\beta, B}(U)$ банахово пространство, состоящее из всех целых аналитических функций на $\mathbb{C}^{k}$ с конечной

1) Проекция $\operatorname{Pr} W$ конуса $W \subset \mathbb{R}^{k}$ есть по определению образ $W \backslash\{0\}$ при каноническом отображении из $\mathbb{R}^{k} \backslash\{0\}$ на сферу $\mathbb{S}_{k-1}=\left(\mathbb{R}^{k} \backslash\{0\}\right) / \mathbb{R}_{+}$; проекция $W$ предполагается открытой в топологии указанной сферы. Отметим, что вырожденный конус $\{0\}$ является конусом с открытой (пустой) проекцией. 
нормой

$$
\|f\|_{U, A, B}=\sup _{z \in \mathbb{C}^{k}}|f(z)| e^{-\rho_{U, A, B}(z)},
$$

где

$$
\rho_{U, A, B}(x+i y)=-\alpha(|x / A|)+\beta(B|y|)+\beta\left(B \delta_{U}(x)\right),
$$

a $\delta_{U}(x)=\inf _{x^{\prime} \in U}\left|x-x^{\prime}\right|-$ расстояние от $x$ до $U$. Пространство $\mathcal{E}_{\alpha}^{\beta}(U)$ определим соотношением $\mathcal{E}_{\alpha}^{\beta}(U)=\bigcup_{A, B>0, W \supset U} \mathcal{E}_{\alpha, A}^{\beta, B}(W)$, где $W$ пробегает по всем коническим окрестностям $U$ и объединение наделяется топологией индуктивного предела.

При $U=\mathbb{R}^{k}$ определение 2 совпадает с определением 1. Везде ниже мы будем предполагать, что все рассматриваемые конусы непусты. Замкнутый конус $K$ называется несущим конусом функционала $u \in \mathcal{E}_{\alpha}^{\prime \beta}\left(\mathbb{R}^{k}\right)$, если $u$ обладает непрерывным продолжением на пространство $\mathcal{E}_{\alpha}^{\beta}(K)$. Следующая теорема представляет собой основной результат данной статьи.

Теорема 1. Пусть функиии $\alpha$ и $\beta$ удовлетворяют условиям определения 1. Если пространство $\mathcal{E}_{\alpha}^{\beta}\left(\mathbb{R}^{k}\right)$ нетривиально (т.е. содержит ненулевые функиии), то имеют место следующие утверждения.

1. Пространство $\mathcal{E}_{\alpha}^{\beta}\left(\mathbb{R}^{k}\right)$ плотно в $\mathcal{E}_{\alpha}^{\beta}(U)$ для любого конуса $U \subset \mathbb{R}^{k}$.

2. Пусть $K_{1}$ и $K_{2}$ - замкнутые конусы в $\mathbb{R}^{k}$. Для любого $и \in \mathcal{E}_{\alpha}^{\prime \beta}\left(\mathbb{R}^{k}\right)$ с несущим конусом $K_{1} \cup K_{2}$ найдутся такие функциональ $u_{1,2} \in \mathcal{E}_{\alpha}^{\prime \beta}\left(\mathbb{R}^{k}\right)$ с несущими конусами $K_{1,2}$, что $u=u_{1}+u_{2}$.

3. Если каждый из конусов $K_{1}$ и $K_{2}$ является несущим для функиионала $и \in$ $\mathcal{E}_{\alpha}^{\prime \beta}\left(\mathbb{R}^{k}\right)$, то несущим будет и конус $K_{1} \cap K_{2}$.

Следует отметить, что аналогичные результаты для пространств ГельфандаШилова $S_{\mu}^{\nu}$ доказывались в работах [6], [7] по-разному для $\nu=0$ и $0<\nu<1$. Подход, используемый в данной статье, позволяет рассматривать оба эти случая единообразно.

Утверждение 1 теоремы 1 показывает, что пространство функционалов с несущим конусом $K$ естественным образом отождествляется с пространством $\mathcal{E}_{\alpha}^{\prime \beta}(K)$. Из определения 2 следует, что

$$
\mathcal{E}_{\alpha}^{\beta}(K)=\bigcup_{W \supset K} \mathcal{E}_{\alpha}^{\beta}(W)
$$

где объединение берется по всем коническим окрестностям $K$ и снабжается топологией индуктивного предела. Согласно утверждению 1 теоремы 1 отсюда вытекает, что $K$ является несущим конусом функционала $u \in \mathcal{E}_{\alpha}^{\prime \beta}\left(\mathbb{R}^{k}\right)$ тогда и только тогда, когда $u$ обладает непрерывным продолжением на пространство $\mathcal{E}_{\alpha}^{\beta}(W)$ для любой конической окрестности $W$ конуса $K$. Из утверждения 3 теоремы 1 следует, что пересечение произвольного семейства $\left\{K_{\omega}\right\}_{\omega \in \Omega}$ несущих конусов функционала $u \in \mathcal{E}_{\alpha}^{\prime \beta}\left(\mathbb{R}^{k}\right)$ снова является несущим конусом $u$. В самом деле, пусть $W$ - коническая окрестность конуса $K=\bigcap_{\omega \in \Omega} K_{\omega}$. Как показывают стандартные соображения компактности (ср. доказательство утверждения А леммы 9 ниже), найдется такое 
конечное семейство $\omega_{1}, \ldots, \omega_{n} \in \Omega$, что $\widetilde{K}=\bigcap_{j=1}^{n} K_{\omega_{j}} \subset W$. Согласно утверждению 3 теоремы 1 конус $\widetilde{K}$ является несущим для $u$, и, следовательно, $u$ обладает непрерывным продолжением на $\mathcal{E}_{\alpha}^{\beta}(W)$. Поэтому конус $K$ является несущим для $u$. В частности, у каждого функционала $u \in \mathcal{E}_{\alpha}^{\prime \beta}\left(\mathbb{R}^{k}\right)$ имеется однозначно определенный минимальный несущий конус - пересечение всех несущих конусов $u$.

Доказательство теоремы 1 существенным образом опирается на использование $L_{2}$-оценок Хермандера для решений неоднородных уравнений Коши-Римана ${ }^{2)}$ $\bar{\partial}_{j} \psi=\eta_{j}, j=1,2, \ldots, k$. Указанные оценки гарантируют существование решения $\psi$, являющегося квадратично-интегрируемым с весом $e^{-\rho} /(1+|z|)^{2}$, при условии, что $\eta_{j}$ квадратично-интегрируемы с весом $e^{-\rho}$, а $\rho$ - плюрисубгармоническая функция на $\mathbb{C}^{k}$. Чтобы проиллюстрировать, как этот результат применяется в нашем случае, мы дадим краткий набросок доказательства утверждения 1.

Пусть $\chi(z)$ - гладкая функция на $\mathbb{C}^{k}$, обращающаяся в нуль при больших $|z|$ и равная единице в окрестности начала координат. Мы построим аппроксимирующую последовательность для $f \in \mathcal{E}_{\alpha}^{\beta}(U)$, полагая $f_{n}(z)=f(z) \chi(z / n)-\psi_{n}(z)$, где члены $\psi_{n}$ вводятся для обеспечения аналитичности $f_{n}$. Это последнее условие означает, что $\psi_{n}$ удовлетворяют уравнениям $\bar{\partial}_{j} \psi_{n}(z)=n^{-1} f(z)\left(\bar{\partial}_{j} \chi\right)(z / n)$. Поэтому можно использовать $L_{2}$-оценки для доказательства того, что $\psi_{n}$ могут быть выбраны настолько малыми, что $f_{n} \in \mathcal{E}_{\alpha}^{\beta}\left(\mathbb{R}^{k}\right)$ и $f_{n} \rightarrow f$ в $\mathcal{E}_{\alpha}^{\beta}(U)$. Однако данная стратегия подразумевает использование норм типа $L_{2}$, в то время как пространства $\mathcal{E}_{\alpha}^{\beta}(U)$ определяются при помощи норм, использующих верхние грани. Эта проблема разрешается в разделе 2 , где выводится эквивалентное представление для $\mathcal{E}_{\alpha}^{\beta}(U)$ в терминах гильбертовых пространств. Другая сложность состоит в том, что весовые функции $e^{-\rho_{U, A, B}}$, фигурирующие в определении 2 , не годятся для $L_{2}$-оценок, поскольку функции $\rho_{U, A, B}$ не являются плюрисубгармоническими. Для преодоления этой трудности в разделе 3 строятся подходящие плюрисубгармонические аппроксимации для $\rho_{U, A, B}$. Доказательство теоремы 1 дано в разделе 4.

\section{2. ПРЕДСТАВЛЕНИЕ $\mathcal{E}_{\alpha}^{\beta}$ ПРИ ПОМОЩИ ГИЛЬБЕРТОВЫХ ПРОСТРАНСТВ}

Пусть $A, B>0$ и $U$ - конус в $\mathbb{R}^{k}$. Обозначим через $H_{\alpha, A}^{\beta, B}(U)$ гильбертово пространство, состоящее из всех целых функций на $\mathbb{C}^{k}$, имеющих конечную норму

$$
\|f\|_{U, A, B}^{\prime}=\left[\int|f(z)|^{2} e^{-2 \rho_{U, A, B}(z)} d \lambda(z)\right]^{1 / 2},
$$

где $d \lambda$ - мера Лебега на $\mathbb{C}^{k}$, а $\rho_{U, A, B}$ определяется формулой (1). Обозначим через $\widetilde{\mathcal{E}}_{\alpha, A}^{\beta, B}(U)$ пространство $\bigcap_{A^{\prime}>A, B^{\prime}>B} \mathcal{E}_{\alpha, A^{\prime}}^{\beta, B^{\prime}}(U)$, снабженное топологией, заданной нормами $\|\cdot\|_{U, A^{\prime}, B^{\prime}}$.

\footnotetext{
2) Здесь и далее мы пользуемся сокращенным обозначением $\bar{\partial}_{j}$ для $\partial / \partial \bar{z}_{j}$.
} 
Лемма 1. Пусть $A, B>0, U-$ конус в $\mathbb{R}^{k}$, а функиии а и $\beta$ удовлетворяют условиям определения 1. Тогда $\widetilde{\mathcal{E}}_{\alpha, A}^{\beta, B}(U)$ является ядерным пространством Фреше, совпадающим (как множество и топологически) $c \bigcap_{A^{\prime}>A, B^{\prime}>B} H_{\alpha, A^{\prime}}^{\beta, B^{\prime}}(U)$.

ДокАЗАТЕЛЬСтво. Пространство $\widetilde{\mathcal{E}}_{\alpha, A}^{\beta, B}(U)$ принадлежит классу пространств $\mathcal{H}(M)$, введенному в работе [11]. Пространство $\mathcal{H}(M)$ (соответственно, $\mathcal{H}_{p}(M)$ при $p \geqslant 1$ ) задается ${ }^{3)}$ при помощи семейства $M=\left\{M_{\gamma}\right\}_{\gamma \in \Gamma}$ строго положительных непрерывных функций на $\mathbb{C}^{k}$ и состоит из всех целых аналитических функций на $\mathbb{C}^{k}$, имеющих конечные нормы

$$
\sup _{z \in \mathbb{C}^{k}} M_{\gamma}(z)|f(z)| \quad\left(\text { соответственно, }\left[\int\left(M_{\gamma}(z)\right)^{p}|f(z)|^{p} d \lambda(z)\right]^{1 / p}\right) .
$$

Предполагается, что: а) для любых $\gamma_{1}, \gamma_{2} \in \Gamma$ найдутся такие $\gamma \in \Gamma$ и $C>0$, что $M_{\gamma} \geqslant C\left(M_{\gamma_{1}}+M_{\gamma_{2}}\right)$; б) существует такое счетное множество $\Gamma^{\prime} \subset \Gamma$, что для любого $\gamma \in \Gamma$ найдутся такие $\gamma^{\prime} \in \Gamma^{\prime}$ и $C>0$, что $C M_{\gamma} \leqslant M_{\gamma^{\prime}}$. Пусть $\Gamma=\left\{\left(A^{\prime}, B^{\prime}\right): A^{\prime}>A\right.$, $\left.B^{\prime}>B\right\}$ и $M_{A^{\prime}, B^{\prime}}(z)=e^{-\rho_{U, A^{\prime}, B^{\prime}}(z)}$. Тогда все приведенные выше условия выполнены, причем $\mathcal{H}(M)=\widetilde{\mathcal{E}}_{\alpha, A}^{\beta, B}(U)$ и $\mathcal{H}_{2}(M)=\bigcap_{A^{\prime}, B^{\prime}} H_{\alpha, A^{\prime}}^{\beta, B^{\prime}}(U)$. Согласно лемме 12 работы [11] $\mathcal{H}(M)$ является ядерным пространством Фреше, совпадающим с $\mathcal{H}_{p}(M)$ для любого $p \geqslant 1$, если выполнены следующие условия.

I. Для любого $\gamma \in \Gamma$ существует такое $\gamma^{\prime} \in \Gamma$, что функция $M_{\gamma}(z) / M_{\gamma^{\prime}}(z)$ суммируема на $\mathbb{C}^{k}$ и стремится к нулю при $|z| \rightarrow \infty$.

II. Для любого $\gamma \in \Gamma$ найдутся такие $\gamma^{\prime} \in \Gamma$, окрестность нуля $\mathcal{B}$ в $\mathbb{C}^{k}$ и $C>0$, что $M_{\gamma}(z) \leqslant C M_{\gamma^{\prime}}(z+\zeta)$ для любых $z \in \mathbb{C}^{k}$ и $\zeta \in \mathcal{B}$.

В рассматриваемом случае выполнение условий I и II вытекает из приводимых ниже лемм 2 и 3 , соответственно. Лемма доказана.

Лемма 2. Пусть $U-$ конус в $\mathbb{R}^{k}$, а функции $\alpha$ и $\beta$ удовлетворяют условиям определения 1. Для любых $A^{\prime}>A>0 u B^{\prime}>B>0$ найдутся такие $\sigma, \tau>0$, что

$$
\rho_{U, A^{\prime}, B^{\prime}}(z)-\rho_{U, A, B}(z)+C \geqslant \sigma|z|^{\tau}, \quad z \in \mathbb{C}^{k},
$$

где $C$ - константа, а $\rho_{U, A, B}$ определяется формулой (1).

ДоКАЗАтЕЛЬСтво. Без потери общности можно считать, что $\beta(0)=0$. Пусть $\varkappa$ удовлетворяет условиям определения 1 , а $s_{0}>0$ таково, что $\alpha\left(s_{0}\right)>0, \beta\left(s_{0}\right)>0$ и функция $\mu(s)=\alpha(s) / s^{\varkappa}$ не убывает при $s \geqslant s_{0}$. При $|x| \geqslant A^{\prime} s_{0}$ имеем

$$
\begin{aligned}
\alpha\left(\frac{|x|}{A}\right)-\alpha\left(\frac{|x|}{A^{\prime}}\right) & =\frac{|x|^{\varkappa}}{A^{\varkappa}} \mu\left(\frac{|x|}{A}\right)-\frac{|x|^{\varkappa}}{A^{\varkappa}} \mu\left(\frac{|x|}{A^{\prime}}\right) \geqslant \\
& \geqslant\left(\frac{1}{A^{\varkappa}}-\frac{1}{A^{\prime \varkappa}}\right) \mu\left(\frac{|x|}{A}\right)|x|^{\varkappa} \geqslant\left(\frac{1}{A^{\varkappa}}-\frac{1}{A^{\prime \varkappa}}\right) \mu\left(s_{0}\right)|x|^{\varkappa} .
\end{aligned}
$$

3) Данное здесь определение $\mathcal{H}(M)$ и $\mathcal{H}_{p}(M)$ является несколько менее общим по сравнению с определением, принятым в [11], но для наших целей его достаточно. 
Поскольку $\beta(0)=0$, выпуклость функции $\beta$ влечет неравенство $\beta(s) \leqslant t \beta(s / t)$ для любых $s \geqslant 0$ и $0<t \leqslant 1$. Отсюда следует, что $\beta(s) / s$ является неубывающей функцией. Поэтому при $|y| \geqslant s_{0} / B^{\prime}$ имеем

$$
\beta\left(B^{\prime}|y|\right)-\beta(B|y|) \geqslant\left(B^{\prime}-B\right) \frac{\beta\left(B^{\prime}|y|\right)}{B^{\prime}|y|}|y| \geqslant\left(B^{\prime}-B\right) \frac{\beta\left(s_{0}\right)}{s_{0}}|y| .
$$

Полагая $\tau=\min (1, \varkappa)$ и складывая оценки для $\alpha$ и $\beta$, находим, что неравенство (3) с $C=0$ справедливо при больших $|z|$, если $\sigma$ достаточно мало. Так как все рассматриваемые функции непрерывны, то, прибавляя к левой части достаточно большую положительную константу, получаем требуемую оценку для всех $z \in \mathbb{C}^{k}$. Лемма доказана.

Лемма 3. Пусть $R>0, U$ - конус в $\mathbb{R}^{k}, \alpha$ и $\beta$ - неубываюшие функиии на $[0, \infty)$, а $\rho_{U, A, B}$ определяется формулой (1). Для любых $A^{\prime}>A>0 u B^{\prime}>B>0$ существует такая константа $C$, что

$$
\rho_{U, A, B}(z+\zeta) \leqslant \rho_{U, A^{\prime}, B^{\prime}}(z)+C, \quad z, \zeta \in \mathbb{C}^{k}, \quad|\zeta| \leqslant R .
$$

ДоКАЗАТЕЛЬСтво. Без потери общности можно считать, что функции $\alpha$ и $\beta$ неотрицательны. Тогда из монотонности $\alpha$ и $\beta$ следует, что $\alpha\left((s+R) / A^{\prime}\right) \leqslant \alpha(s / A)+$ $\alpha\left(R /\left(A^{\prime}-A\right)\right)$ и $\beta(B(s+R)) \leqslant \beta\left(B^{\prime} s\right)+\beta\left(R B B^{\prime} /\left(B^{\prime}-B\right)\right)$. Пусть $z=x+i y$ и $\zeta=\xi+i \eta$ таковы, что $|\zeta| \leqslant R$. Так как $\delta_{U}(x+\xi) \leqslant \delta_{U}(x)+|\xi|$, то

$$
\begin{gathered}
\alpha\left(\frac{|x|}{A^{\prime}}\right) \leqslant \alpha\left(\frac{|x+\xi|+R}{A^{\prime}}\right) \leqslant \alpha\left(\frac{|x+\xi|}{A}\right)+\alpha\left(\frac{R}{A^{\prime}-A}\right), \\
\beta(B|y+\eta|)+\beta\left(B \delta_{U}(x+\xi)\right) \leqslant \beta\left(B^{\prime}|y|\right)+\beta\left(B^{\prime} \delta_{U}(x)\right)+2 \beta\left(\frac{R B B^{\prime}}{B^{\prime}-B}\right) .
\end{gathered}
$$

Складывая эти неравенства, получаем требуемую оценку.

СлеДСТвиЕ 1. Если $f \in \mathcal{E}_{\alpha}^{\beta}(U), \operatorname{mo} f(\cdot+\zeta) \in \mathcal{E}_{\alpha}^{\beta}(U)$ для любого $\zeta \in \mathbb{C}^{k}$.

Напомним, что пространства DFS (dual Fréchet-Schwartz) являются по определению индуктивными пределами последовательностей локально-выпуклых пространств с инъективными компактными связывающими отображениями (см. [12]).

Лемма 4. Пусть $U-$ конус в $\mathbb{R}^{k}$, а функиии $\alpha$ и $\beta$ удовлетворяют условиям определения 1. Тогда $\mathcal{E}_{\alpha}^{\beta}(U)$ - ядерное пространство DFS, совпадающее (как множество и топологччески) с пространством

$$
\bigcup_{A, B>0, W \supset U} H_{\alpha, A}^{\beta, B}(W)
$$

где $W$ пробегает по всем коническим окрестностям конуса $U$, а обгединение наделяется топологией индуктивного предела. 
ДоказАтельство. Пусть $A^{\prime}>A>0, B^{\prime}>B>0$, а $W \supset W^{\prime}$ - конические окрестности $U$. Тогда имеются непрерывные вложения $\mathcal{E}_{\alpha, A}^{\beta, B}(W) \rightarrow \widetilde{\mathcal{E}}_{\alpha, A}^{\beta, B}(W) \rightarrow$ $\mathcal{E}_{\alpha, A^{\prime}}^{\beta, B^{\prime}}\left(W^{\prime}\right)$, и, следовательно,

$$
\mathcal{E}_{\alpha}^{\beta}(U)=\bigcup_{A, B>0, W \supset U} \widetilde{\mathcal{E}}_{\alpha, A}^{\beta, B}(W)
$$

Поскольку счетные индуктивные пределы ядерных пространств являются ядерными пространствами (см., например, [13], следствие теоремы III.7.4), ядерность пространства $\mathcal{E}_{\alpha}^{\beta}(U)$ вытекает из леммы 1. Так как все непрерывные отображения из ядерных пространств в банаховы пространства являются ядерными ([13], теорема III.7.2), то вложение $\mathcal{E}_{\alpha, A}^{\beta, B}(W) \rightarrow \mathcal{E}_{\alpha, A^{\prime}}^{\beta, B^{\prime}}\left(W^{\prime}\right)$ является ядерным как композиция ядерного и непрерывного отображений. Отсюда следует, что $\mathcal{E}_{\alpha}^{\beta}(U)$ - пространство DFS, поскольку ядерные отображения компактны ([13], следствие 1 теоремы III.7.1). В силу леммы 1 имеются непрерывные вложения $H_{\alpha, A}^{\beta, B}(W) \rightarrow \widetilde{\mathcal{E}}_{\alpha, A}^{\beta, B}(W) \rightarrow H_{\alpha, A^{\prime}}^{\beta, B^{\prime}}\left(W^{\prime}\right)$. Согласно (4) отсюда следует, что $\mathcal{E}_{\alpha}^{\beta}(U)=\bigcup_{A, B>0, W \supset U} H_{\alpha, A}^{\beta, B}(W)$. Лемма доказана.

\section{3. ПЛЮРИСУБГАРМОНИЧЕСКИЕ АППРОКСИМАЦИИ}

Напомним, что норма $|\cdot|$ предполагается равномерной.

Tеорема 2. Пусть $A, B>0, U$ - непустой конус в $\mathbb{R}^{k}, \alpha$ - непрерывная неубъвающая функиия на $[0, \infty)$, а $\beta$ - непрерывная выпуклая неубывающая функиия на $[0, \infty)$. Предположим, что существует такая не равная тождественно нулю целая функиия ч на $\mathbb{C}$, что

$$
|\varphi(z)| \leqslant e^{\beta\left(\left|B_{0} y\right|\right)-\alpha\left(\left|x / A_{0}\right|\right)}, \quad z=x+i y \in \mathbb{C},
$$

при некоторьх $A_{0}, B_{0}>0$. Тогда для любого $R>0$ найдется такая плюрисубгармоническая функция $\rho_{R}$ на $\mathbb{C}^{k}$, что

$$
\begin{aligned}
& \rho_{R}(z) \leqslant \rho_{\mathbb{R}^{k}, A^{\prime}, B^{\prime}}(z)+\beta(2 B e R), \quad z=x+i y \in \mathbb{C}^{k}, \\
& \rho_{R}(z) \leqslant \rho_{U, A^{\prime}, B^{\prime}}(z), \quad z \in \mathbb{C}^{k}, \\
& \rho_{R}(z) \geqslant \rho_{U, A, B}(z)-H, \quad|x| \leqslant R,
\end{aligned}
$$

где $\rho_{U, A, B}$ определяется формулой (1), $H$ - не зависящая от $R$ константа, $A^{\prime}=2 A$ и $B^{\prime}=(2 e k+1) B+4 k A_{0} B_{0} / A$. Если бункция $\alpha$ вогнута, то можно положить $A^{\prime}=A$.

СлЕДСТВИЕ 2. Если выполнены условия теоремы 2, то существует такая плюрисубгармоническая функиия $\rho$, что

$$
\rho_{U, A, B}(z)-H \leqslant \rho(z) \leqslant \rho_{U, A^{\prime}, B^{\prime}}(z), \quad z \in \mathbb{C}^{k},
$$

где $\rho_{U, A, B}$ дается формулой (1), $H$ - константа, $A^{\prime}=2 A$ u $B^{\prime}=(2 e k+1) B+$ $4 k A_{0} B_{0} / A$. Если функция $\alpha$ вогнута, то можно положить $A^{\prime}=A$. 
ДокАЗАТЕЛЬСтво. Пусть $\rho_{R}$ удовлетворяет условиям теоремы 2. Тогда функция $\rho(z)=\varlimsup_{z^{\prime} \rightarrow z} \sup _{R>0} \rho_{R}\left(z^{\prime}\right)$ является плюрисубгармонической ([14], раздел II.10.3) и удовлетворяет требуемой оценке.

Для доказательства теоремы 2 нам потребуются две леммы.

Лемма 5. Пусть $\alpha$ и $\beta$ - непрерьвные неубывающие функиии на $[0, \infty)$ и существует не равная тождественно нулю целая аналитическая функиия $\varphi$ на $\mathbb{C}$, удовлетворяющая оценке

$$
|\varphi(z)| \leqslant e^{\beta(|y|)-\alpha(|x|)}, \quad z=x+i y \in \mathbb{C} .
$$

Тогда найдутся такие плюрисубгармоническая функиия $\rho$ на $\mathbb{C}^{k}$ и константа $H$, что

$$
-\alpha(2|x|)-k \beta(4|y|)-H \leqslant \rho(z) \leqslant k \beta(4|y|)-\alpha(|x|), \quad z=x+i y \in \mathbb{C}^{k} .
$$

Если функиия $\alpha$ вогнута, то существуют такие плюрисубгармоническая функиия $\rho$ на $\mathbb{C}^{k}$ и константа $H$, что

$$
-\alpha(|x|)-k \beta(2|y|)-H \leqslant \rho(z) \leqslant k \beta(2|y|)-\alpha(|x|), \quad z=x+i y \in \mathbb{C}^{k} .
$$

ДокАЗАТЕЛЬСтво. Без потери общности можно считать, что $\alpha(0)=\beta(0)=0$ и $\varphi(0) \neq 0$ (если $\varphi$ имеет нуль порядка $n$ при $z=0$, то можно заменить $\varphi$ на $\tilde{\varphi}(z)=C \varphi(z) / z^{n}$; если $C$ достаточно мало, то $\tilde{\varphi}$ удовлетворяет (7)). Положим

$$
\begin{aligned}
\tilde{\rho}(z) & =\sup _{\zeta \in \mathbb{C}^{k}}\{\Phi(z-\zeta)+M(\zeta)\}, \\
M(\zeta) & =\inf _{z^{\prime}=x^{\prime}+i y^{\prime} \in \mathbb{C}^{k}}\left\{-\Phi\left(z^{\prime}-\zeta\right)+k \beta\left(4\left|y^{\prime}\right|\right)-\alpha\left(\left|x^{\prime}\right|\right)\right\},
\end{aligned}
$$

где $\Phi(z)=\sum_{j=1}^{k} \ln \left|\varphi\left(2 z_{j}\right)\right|$. Очевидно, имеем $\tilde{\rho}(z) \leqslant k \beta(4|y|)-\alpha(|x|)$. Так как $\Phi-$ плюрисубгармоническая функция, то функция $\rho(z)=\varlimsup_{z^{\prime} \rightarrow z} \tilde{\rho}(z)$ также является плюрисубгармонической (см. [14], раздел II.10.3). Ввиду непрерывности функций $\alpha$ и $\beta$ имеем $\tilde{\rho}(z) \leqslant \rho(z) \leqslant k \beta(4|y|)-\alpha(|x|)$, и нам остается показать, что $\tilde{\rho}(z) \geqslant$ $-\alpha(2|x|)-k \beta(4|y|)-H$. Из (7) следует, что

$$
-\Phi\left(z^{\prime}-z\right) \geqslant \alpha\left(2\left|x^{\prime}-x\right|\right)-k \beta\left(2\left|y^{\prime}-y\right|\right), \quad \zeta=\xi+i \eta,
$$

и, полагая $H=-\Phi(0)=-k \ln |\varphi(0)|$, получаем

$$
\tilde{\rho}(z) \geqslant-H+M(z) \geqslant \inf _{x^{\prime}, y^{\prime} \in \mathbb{R}^{k}}\left\{k \beta\left(4\left|y^{\prime}\right|\right)-k \beta\left(2\left|y^{\prime}-y\right|\right)+\alpha\left(2\left|x^{\prime}-x\right|\right)-\alpha\left(\left|x^{\prime}\right|\right)\right\}-H .
$$

Поскольку обе функции $\alpha$ и $\beta$ неотрицательны и монотонны, имеем

$$
\beta\left(2\left|y^{\prime}\right|\right)-\beta\left(\left|y^{\prime}-y\right|\right) \geqslant-\beta(2|y|), \quad \alpha\left(2\left|x^{\prime}-x\right|\right)-\alpha\left(\left|x^{\prime}\right|\right) \geqslant-\alpha(2|x|) .
$$

Подставляя эти неравенства в (11), получаем требуемую оценку снизу для $\tilde{\rho}$. Таким образом, формула (8) доказана. 
Пусть теперь функция $\alpha$ вогнута. Заменим $\beta\left(4\left|y^{\prime}\right|\right)$ на $\beta\left(2\left|y^{\prime}\right|\right)$ в определении $M(\zeta)(10)$ и модифицируем $\Phi(z)$, полагая $\Phi(z)=\sum_{j=1}^{k} \ln \left|\varphi\left(z_{j}\right)\right|$. Определяя $\tilde{\rho}$ и $\rho$ так же, как и выше, получаем $\tilde{\rho}(z) \leqslant \rho(z) \leqslant k \beta(2|y|)-\alpha(|x|)$. Повторяя приведенные выше рассуждения, приходим к оценке

$$
\tilde{\rho}(z) \geqslant \inf _{x^{\prime}, y^{\prime} \in \mathbb{R}^{k}}\left\{k \beta\left(2\left|y^{\prime}\right|\right)-k \beta\left(\left|y^{\prime}-y\right|\right)+\alpha\left(\left|x^{\prime}-x\right|\right)-\alpha\left(\left|x^{\prime}\right|\right)\right\}-H .
$$

Так как функция $\alpha$ вогнута и $\alpha(0)=0$, то $\alpha(s+t) \leqslant \alpha(s)+\alpha(t)$ для любых $s, t \geqslant 0$. Отсюда следует, что $\alpha\left(\left|x+x^{\prime}\right|\right) \leqslant \alpha(|x|)+\alpha\left(\left|x^{\prime}\right|\right)$ для любых $x, x^{\prime} \in \mathbb{R}^{k}$. Делая замену $x^{\prime} \rightarrow x^{\prime}-x$, находим, что $\alpha\left(\left|x^{\prime}-x\right|\right)-\alpha\left(\left|x^{\prime}\right|\right) \geqslant-\alpha(|x|)$. Подставляя эту оценку и первое из неравенств (12) в (13), получаем $\tilde{\rho}(z) \geqslant-\alpha(|x|)-k \beta(2|y|)-H$, что завершает доказательство (9).

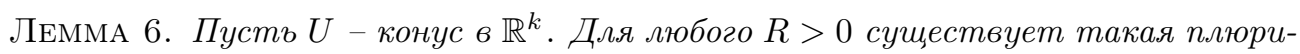
субгармоническая функция $\sigma_{R}$ на $\mathbb{C}^{k}$, что

$$
\begin{aligned}
& \sigma_{R}(z) \leqslant k|y|+R, \quad z=x+i y \in \mathbb{C}^{k}, \\
& \sigma_{R}(z) \leqslant k|y|+\delta_{U}(x), \quad z \in \mathbb{C}^{k}, \\
& \sigma_{R}(z) \geqslant \delta_{U}\left(\frac{x}{e}\right), \quad|x| \leqslant R,
\end{aligned}
$$

где $\delta_{U}(x)=\inf _{x^{\prime} \in U}\left|x-x^{\prime}\right|-$ расстояние от $x$ до $U$.

ДокАЗАТЕЛЬСтво. Для любого $a>0$ определим субгармоническую функцию $\Theta_{a}$ на $\mathbb{C}$ :

$$
\Theta_{a}(z)=a \ln \left|\frac{\sin (z / a)}{z / a}\right| .
$$

Эта функция удовлетворяет неравенствам

$$
\begin{gathered}
\Theta_{a}(i y) \geqslant 0, \quad y \in \mathbb{R}, \\
\Theta_{a}(z) \leqslant|y|-a \ln ^{+}\left(\frac{|x|}{a}\right), \quad z=x+i y \in \mathbb{C},
\end{gathered}
$$

где $\ln ^{+}(r)=\max (\ln r, 0)$. В самом деле, поскольку

$$
\Theta_{a}(i y)=a \ln \left(\frac{\operatorname{sh}(y / a)}{y / a}\right)
$$

оценка (17) вытекает из неравенства $\operatorname{sh} y / y \geqslant 1, y \in \mathbb{R}$. Далее, из неравенств

$$
|\sin z| \leqslant e^{|y|}, \quad\left|\frac{\sin z}{z}\right| \leqslant e^{|y|}, \quad z=x+i y \in \mathbb{C},
$$

следует, что

$$
\left|\frac{\sin (z / a)}{z / a}\right| \leqslant e^{|y / a|} \min (1, a /|x|)
$$


и, переходя к логарифмам, получаем (18). Положим теперь

$$
\begin{aligned}
& \tilde{\sigma}_{R}(z)=\sup _{a>0, \xi \in \mathbb{R}^{k},|\xi| \leqslant R}\left\{\Phi_{a}(z-\xi)+M_{a}(\xi)\right\}, \\
& M_{a}(\xi)=\inf _{z^{\prime}=x^{\prime}+i y^{\prime} \in \mathbb{C}^{k}}\left\{-\Phi_{a}\left(z^{\prime}-\xi\right)+k\left|y^{\prime}\right|+\delta_{U}\left(x^{\prime}\right)\right\},
\end{aligned}
$$

где $\Phi_{a}(z)=\sum_{j=1}^{k} \Theta_{a}\left(z_{j}\right)$. Очевидно, $\tilde{\sigma}_{R}(z) \leqslant k|y|+\delta_{U}(x)$. Согласно неравенству (18) имеем $\Phi_{a}(z-\xi) \leqslant k|y|$ и, следовательно, $\tilde{\sigma}_{R}(z) \leqslant k|y|+\sup _{a>0,|\xi| \leqslant R} M_{a}(\xi)$. Поскольку $\Phi_{a}(0)=0$, из определения $M_{a}$ вытекает, что $M_{a}(\xi) \leqslant \delta_{U}(\xi)$. Поэтому $\tilde{\sigma}_{R}(z) \leqslant$ $k|y|+R$. Так как $\Phi_{a}-$ плюрисубгармонические функции, то $\sigma_{R}(z)=\varlimsup_{z^{\prime} \rightarrow z} \tilde{\sigma}_{R}(z)-$ также плюрисубгармоническая функция, а из непрерывности функций $\delta_{U}(x)$ и $|y|$ следует, что $\sigma_{R}$ удовлетворяет (14) и (15). Из оценки (17) вытекает, что $\Phi_{a}(i y) \geqslant 0$, $y \in \mathbb{R}^{k}$. Следовательно,

$$
\tilde{\sigma}_{R}(z) \geqslant \sup _{a>0}\left(\Phi_{a}(i y)+M_{a}(x)\right) \geqslant \sup _{a>0} M_{a}(x), \quad|x| \leqslant R .
$$

С помощью элементарных неравенств $\sum_{j=1}^{k} \ln ^{+}\left|x_{j}\right| \geqslant \ln ^{+}(|x|)$ и $\sum_{j=1}^{k}\left|y_{j}\right| \leqslant k|y|$ из оценки (18) получаем соотношение

$$
M_{a}(x) \geqslant \inf _{x^{\prime} \in \mathbb{R}^{k}}\left\{a \ln ^{+}\left(\frac{\left|x^{\prime}\right|}{a}\right)+\delta_{U}\left(x+x^{\prime}\right)\right\} .
$$

Оценивая $\delta_{U}\left(x+x^{\prime}\right)$ снизу посредством $\max \left(\delta_{U}(x)-\left|x^{\prime}\right|, 0\right)$ и вычисляя нижнюю грань по $x^{\prime}$, получаем $M_{a}(x) \geqslant a \ln ^{+}\left(\delta_{U}(x) / a\right)$. Пусть $\delta_{U}(x)>0$ и $a_{0}=\delta_{U}(x) / e$. Ввиду (19) находим

$$
\tilde{\sigma}_{R}(z) \geqslant M_{a_{0}}(x) \geqslant \delta_{U}(x) / e, \quad|x| \leqslant R .
$$

Если $\delta_{U}(x)=0$ и $|x| \leqslant R$, то оценка $\tilde{\sigma}_{R}(z) \geqslant \delta_{U}(x) / e$ также верна, так как ввиду (19) и $(20)$ имеем $\tilde{\sigma}_{R}(z) \geqslant 0$. Отсюда следует неравенство (16), поскольку $\sigma_{R} \geqslant \tilde{\sigma}_{R}$. Лемма доказана.

ДОКАЗАТЕЛЬСТво ТЕОРЕМЫ 2. Без потери общности можно считать, что $\beta(0)=0$. Положим $\rho_{R}^{\prime}(z)=\beta\left(B e \sigma_{R}(z)\right)$, где $\sigma_{R}$ - плюрисубгармоническая функция, удовлетворяющая условиям леммы 6 . Так как композиция неубывающей выпуклой функции с плюрисубгармонической функцией является плюрисубгармонической функцией (см. [15], теорема 3.2.18 и раздел 4.1), то $\rho_{R}^{\prime}$ - плюрисубгармоническая функция. Ввиду монотонности $\beta$ неравенства (14)-(16) влекут оценки

$$
\begin{aligned}
& \rho_{R}^{\prime}(z) \leqslant \beta(2 B e k|y|)+\beta(2 B e R), \quad z=x+i y \in \mathbb{C}^{k}, \\
& \rho_{R}^{\prime}(z) \leqslant \beta(2 B e k|y|)+\beta\left(2 B e \delta_{U}(x)\right), \quad z \in \mathbb{C}^{k}, \\
& \rho_{R}^{\prime}(z) \geqslant \beta\left(\delta_{U}(B x)\right), \quad|x| \leqslant R .
\end{aligned}
$$

В силу леммы 5 существуют такие плюрисубгармоническая функция $\rho^{\prime \prime}$ и константа $H$, что

$$
-\alpha(|x / A|)-k \beta(D|y|)-H \leqslant \rho^{\prime \prime}(z) \leqslant k \beta(D|y|)-\alpha\left(\left|x / A^{\prime}\right|\right),
$$


где $D=2 A_{0} B_{0} / A$ и $A^{\prime}=2 A\left(A^{\prime}=A\right.$, если функция $\alpha$ вогнута). Положим $\rho_{R}(z)=\rho_{R}^{\prime}(z)+\rho^{\prime \prime}(z)+k \beta(D|y|)+\beta(|B y|)$. Функция $\rho_{R}$ является плюрисубгармонической, поскольку $\beta(D|y|)$ и $\beta(|B y|)$ - выпуклые и, следовательно, плюрисубгармонические функции. Оценки (6) с $B^{\prime}=2 k D+(2 e k+1) B$ легко следуют из (22), (23) и неравенства

$$
2 k \beta(D|y|)+\beta(B|y|)+\beta(2 B e k|y|) \leqslant \beta\left(B^{\prime}|y|\right),
$$

вытекающего из выпуклости $\beta$ и условия $\beta(0)=0$.

\section{4. ДОКАЗАТЕЛЬСТВО ТЕОРЕМЫ 1}

Как и выше, будем обозначать через $d \lambda$ меру Лебега на $\mathbb{C}^{k}$. Доказательство теоремы 1 опирается на следующее утверждение, являющееся частным случаем теоремы 4.2.6 книги [15].

Лемма 7. Пусть $\rho$ - плюрисубгармоническая функция на $\mathbb{C}^{k} u \eta_{j}, j=1,2, \ldots, k$, - локально квадратично-интегрируемые функции на $\mathbb{C}^{k}$. Предположим, что

$$
\int\left|\eta_{j}(z)\right|^{2} e^{-\rho(z)} d \lambda(z)<\infty
$$

для всех $ј$ и что $\eta_{j}$ удовлетворяют (как обобщенные функиии) условиям согласования $\bar{\partial}_{j} \eta_{l}=\bar{\partial}_{l} \eta_{j}$. Тогда у неоднороднъх уравнений Коши-Римана $\bar{\partial}_{j} \psi=\eta_{j}$ имеется локально квадратично-интегрируемое решение, удовлетворяющее оченке ${ }^{4)}$

$$
2 \int|\psi(z)|^{2} e^{-\rho(z)}\left(1+|z|^{2}\right)^{-2} d \lambda(z) \leqslant k^{2} \sum_{j=1}^{k} \int\left|\eta_{j}(z)\right|^{2} e^{-\rho(z)} d \lambda(z) .
$$

Пусть $\rho$ - измеримая локально ограниченная функция на $\mathbb{C}^{k}$. Обозначим через $L_{2}\left(\mathbb{C}^{k}, e^{-\rho} d \lambda\right)$ гильбертово пространство функций, квадратично-интегрируемых по мере $e^{-\rho} d \lambda$, а через $H_{\rho}$ - замкнутое подпространство $L_{2}\left(\mathbb{C}^{k}, e^{-\rho} d \lambda\right)$, состоящее из целых аналитических функций.

Лемма 8. Пусть $\rho_{0}, \rho$ и $\rho^{\prime}-$ такие измеримые локально ограниченнъе функиии на $\mathbb{C}^{k}$, что $\rho_{0} \leqslant \rho^{\prime}$ u $\rho \leqslant \rho^{\prime}$. Предположим, что для любого $R>0$ существует такая плюрисубгармоническая функиия $\rho_{R}$, что

$$
\begin{gathered}
\rho_{R}(z)+2 \ln \left(1+|z|^{2}\right) \leqslant \rho^{\prime}(z), \quad z \in \mathbb{C}^{k}, \\
\rho_{R}(z)+2 \ln \left(1+|z|^{2}\right) \leqslant \rho_{0}(z)+C_{R}, \quad z \in \mathbb{C}^{k}, \\
\rho_{R}(z) \geqslant \rho(z), \quad|z| \leqslant R,
\end{gathered}
$$

где $C_{R}$ - константа. Тогда $H_{\rho}$ содержится в замыкании $H_{\rho_{0}}$ в $H_{\rho^{\prime}}$.

\footnotetext{
4) Оценка в лемме 7 отличается от оценки в [15] множителем $k^{2}$ в правой части, возникающим из-за использования нами равномерной нормы вместо применявшейся в [15] евклидовой нормы.
} 
ДоказАтельство. Пусть $f \in H_{\rho}$ и $\chi$ - такая гладкая функция на $\mathbb{C}^{k}$, что $0 \leqslant$ $\chi \leqslant 1, \chi(z)=1$ при $|z| \leqslant 1$ и $\chi(z)=0$ при $|z| \geqslant 2$. Положим $g_{n}(z)=f(z) \chi(z / n)$. Так как $\bar{\partial}_{j} g_{n}(z)=n^{-1} f(z)\left(\bar{\partial}_{j} \chi\right)(z / n)$ обращается в нуль при $|z| \geqslant 2 n$, то из (26) следует, что

$$
\int\left|\bar{\partial}_{j} g_{n}(z)\right|^{2} e^{-\rho_{2 n}(z)} d \lambda(z) \leqslant \int\left|\bar{\partial}_{j} g_{n}(z)\right|^{2} e^{-\rho(z)} d \lambda(z) \leqslant \frac{a}{n^{2}}\|f\|_{\rho}^{2}, \quad j=1,2, \ldots, k,
$$

где $\|\cdot\|_{\rho}-$ норма в $L_{2}\left(\mathbb{C}^{k}, e^{-\rho} d \lambda\right)$ и $a=\sup _{z, j}\left|\bar{\partial}_{j} \chi(z)\right|^{2}$. В силу леммы 7 существует такая локально квадратично-интегрируемая функция $\psi_{n}$ на $\mathbb{C}^{k}$, что $\bar{\partial}_{j} \psi_{n}=\bar{\partial}_{j} g_{n}$ и

$$
\int\left|\psi_{n}\right|^{2} e^{-\rho_{2 n}(z)}\left(1+|z|^{2}\right)^{-2} d \lambda(z) \leqslant \frac{k^{3} a}{2 n^{2}}\|f\|_{\rho}^{2} .
$$

С учетом (25) отсюда вытекает, что $\left\|\psi_{n}\right\|_{\rho_{0}}<\infty$. Далее, имеем $\bar{\partial}_{j}\left(g_{n}-\psi_{n}\right)=0$ и, следовательно, $g_{n}-\psi_{n}$ совпадает почти везде с некоторой целой аналитической функцией $f_{n}$. Так как $g_{n}$ - функция с компактным носителем, то $\left\|g_{n}\right\|_{\rho_{0}}<\infty$ и, следовательно, $f_{n} \in H_{\rho_{0}}$. Поскольку $\rho \leqslant \rho^{\prime}$, имеем

$$
\left\|f-g_{n}\right\|_{\rho^{\prime}}^{2} \leqslant \int_{|z| \geqslant n}|f(z)|^{2} e^{-\rho(z)} d \lambda(z) .
$$

Поэтому $g_{n} \rightarrow f$ в $L_{2}\left(\mathbb{C}^{k}, e^{-\rho^{\prime}} d \lambda\right)$. В силу (24) и $(27)$ имеем $\left\|\psi_{n}\right\|_{\rho^{\prime}}^{2} \leqslant k^{3} a\|f\|_{\rho}^{2} /\left(2 n^{2}\right)$. Таким образом, $f_{n} \rightarrow f$ в $H_{\rho^{\prime}}$ и лемма доказана.

Лемма 8 была доказана в работе [7] другим способом при дополнительном предположении о гладкости $\rho_{R}$. Приведенное здесь простое доказательство ближе к способу рассуждения, намеченному в разделе 5 работы [6].

Заметим, что рассматривавшиеся в разделе 2 пространства $H_{\alpha, A}^{\beta, B}(U)$ совпадают с $H_{\rho}$ при $\rho=2 \rho_{U, A, B}$.

\section{ДОКАЗАТЕЛЬСТВО ТЕОРЕМЫ 1.}

1. Пусть $f \in \mathcal{E}_{\alpha}^{\beta}(U)$. В силу леммы 4 существуют такие $A, B>0$ и коническая окрестность $W$ конуса $U$, что $f \in H_{\alpha, A}^{\beta, B}(W)$. Ввиду следствия 1 нетривиальность $\mathcal{E}_{\alpha}^{\beta}\left(\mathbb{R}^{k}\right)$ влечет существование таких $A_{0}, B_{0}>0$ и $f_{0} \in \mathcal{E}_{\alpha, A_{0}}^{\beta, B_{0}}\left(\mathbb{R}^{k}\right)$, что $f_{0}(0) \neq 0$ и $\left\|f_{0}\right\|_{\mathbb{R}^{k}, A_{0}, B_{0}} \leqslant 1$. Тогда целая функция $\varphi(z)=f_{0}(z, 0, \ldots, 0)$ на $\mathbb{C}$ не равна тождественно нулю и удовлетворяет условию (5). Из леммы 2 и теоремы 2 следует, что функции $\rho=2 \rho_{W, A, B}-H, \rho_{0}=2 \rho_{\mathbb{R}^{k}, A^{\prime}, B^{\prime}}$ и $\rho^{\prime}=2 \rho_{W, A^{\prime}, B^{\prime}}$ удовлетворяют условиям леммы 8 , если $A^{\prime}>2 A, B^{\prime}>(2 e k+1) B+4 k A_{0} B_{0} / A$ и константа $H$ достаточно велика. В силу леммы 8 найдется последовательность $f_{n} \in H_{\alpha, A^{\prime}}^{\beta, B^{\prime}}\left(\mathbb{R}^{k}\right)$, сходящаяся к $f$ в $H_{\alpha, A^{\prime}}^{\beta, B^{\prime}}(W)$. Согласно лемме 4 гильбертова топология пространства $H_{\alpha, A^{\prime}}^{\beta, B^{\prime}}(W)$ сильнее, чем топология, индуцированная из $\mathcal{E}_{\alpha}^{\beta}(U)$. Следовательно, $f_{n} \rightarrow f$ в $\mathcal{E}_{\alpha}^{\beta}(U)$.

2. Пусть $l: \mathcal{E}_{\alpha}^{\beta}\left(K_{1} \cup K_{2}\right) \rightarrow \mathcal{E}_{\alpha}^{\beta}\left(K_{1}\right) \oplus \mathcal{E}_{\alpha}^{\beta}\left(K_{2}\right)$ и $m: \mathcal{E}_{\alpha}^{\beta}\left(K_{1}\right) \oplus \mathcal{E}_{\alpha}^{\beta}\left(K_{2}\right) \rightarrow \mathcal{E}_{\alpha}^{\beta}\left(K_{1} \cap K_{2}\right)-$ непрерывные линейные отображения, переводящие $f$ в $(f, f)$ и $\left(f_{1}, f_{2}\right)$ в $f_{1}-f_{2}$, соответственно. Образ отображения $l$ замкнут, так как в силу определения 2 справедливо равенство $\mathcal{E}_{\alpha}^{\beta}\left(K_{1}\right) \cap \mathcal{E}_{\alpha}^{\beta}\left(K_{2}\right)=\mathcal{E}_{\alpha}^{\beta}\left(K_{1} \cup K_{2}\right)$ и, следовательно, $\operatorname{Im} l=\operatorname{Ker} m$. 
В силу леммы 4 отсюда вытекает, что $\operatorname{Im} l$ является пространством $\mathrm{DFS}^{5)}$. Пусть $u \in \mathcal{E}_{\alpha}^{\prime \beta}\left(\mathbb{R}^{k}\right)$ - функционал с несущим конусом $K_{1} \cup K_{2}, \hat{u}$ - его непрерывное продолжение на $\mathcal{E}_{\alpha}^{\beta}\left(K_{1} \cup K_{2}\right)$. Согласно теореме об открытом отображении (см. [13], теорема IV.8.3; эта теорема применима, поскольку пространства DFS являются В-полными как сильные сопряженные рефлексивных пространств Фреше [12]) линейный функционал $\hat{u} l^{-1}$ непрерывен на $\operatorname{Im} l$ и по теореме Хана-Банаха обладает непрерывным продолжением $v$ на все пространство $\mathcal{E}_{\alpha}^{\beta}\left(K_{1}\right) \oplus \mathcal{E}_{\alpha}^{\beta}\left(K_{2}\right)$. Пусть $v_{1}$ и $v_{2}$ - ограничения $v$ на $\mathcal{E}_{\alpha}^{\beta}\left(K_{1}\right)$ и $\mathcal{E}_{\alpha}^{\beta}\left(K_{2}\right)$, соответственно. Тогда для любого $f \in \mathcal{E}_{\alpha}^{\beta}\left(K_{1} \cup K_{2}\right)$ имеем $\hat{u}(f)=v(f, f)=v_{1}(f)+v_{2}(f)$. Это означает, что $u=u_{1}+u_{2}$, где $u_{1,2}$ - ограничения $v_{1,2}$ на $\mathcal{E}_{\alpha}^{\beta}\left(\mathbb{R}^{k}\right)$. По построению конусы $K_{1,2}$ являются несущими для $u_{1,2}$.

3. Пусть отображения $l$ и $m$ определены, как и выше, $u \in \mathcal{E}_{\alpha}^{\prime \beta}\left(\mathbb{R}^{k}\right)-$ функционал, для которого оба конуса $K_{1}$ и $K_{2}$ являются несущими, и $u_{1,2}$ - его непрерывные продолжения на $\mathcal{E}_{\alpha}^{\prime \beta}\left(K_{1,2}\right)$. Предположим, что отображение $m$ сюръективно. Тогда из теоремы об открытом отображении следует, что пространство $\mathcal{E}_{\alpha}^{\beta}\left(K_{1} \cap K_{2}\right)$ топологически изоморфно факторпространству $\left(\mathcal{E}_{\alpha}^{\beta}\left(K_{1}\right) \oplus \mathcal{E}_{\alpha}^{\beta}\left(K_{2}\right)\right) /$ Ker $m$. Определим непрерывный линейный функционал $v$ на $\mathcal{E}_{\alpha}^{\beta}\left(K_{1}\right) \oplus \mathcal{E}_{\alpha}^{\beta}\left(K_{2}\right)$ соотношением $v\left(f_{1}, f_{2}\right)=u_{1}\left(f_{1}\right)-u_{2}\left(f_{2}\right)$. В силу утверждения 1 теоремы функционалы $u_{1}$ и $u_{2}$ совпадают на $\mathcal{E}_{\alpha}^{\beta}\left(K_{1} \cup K_{2}\right)$, и, следовательно, Ker $v \supset \operatorname{Im} l$. Tак как $\operatorname{Ker} m=\operatorname{Im} l$, то это включение влечет существование такого функционала $\hat{u} \in \mathcal{E}_{\alpha}^{\prime \beta}\left(K_{1} \cap K_{2}\right)$, что $v=\hat{u} m$. Если $f_{1,2} \in \mathcal{E}_{\alpha}^{\beta}\left(K_{1,2}\right)$, то $\hat{u}\left(f_{1}\right)=v\left(f_{1}, 0\right)=u_{1}\left(f_{1}\right)$ и $\hat{u}\left(f_{2}\right)=v\left(0,-f_{2}\right)=u_{2}\left(f_{2}\right)$. Поэтому $\hat{u}$ служит непрерывным продолжением $u$ на $\mathcal{E}_{\alpha}^{\beta}\left(K_{1} \cap K_{2}\right)$. Таким образом, доказательство утверждения 3 теоремы сводится к доказательству сюръективности $m$. Последняя вытекает из следующего результата о разложении пробных функций.

Tеорема 3. Пусть пространство $\mathcal{E}_{\alpha}^{\beta}\left(\mathbb{R}^{k}\right)$ нетривиально, $K_{1}$ и $K_{2}$ - замкнутые конусъ в $\mathbb{R}^{k}$ и $f \in \mathcal{E}_{\alpha}^{\beta}\left(K_{1} \cap K_{2}\right)$. Тогда существуют такие $f_{1,2} \in \mathcal{E}_{\alpha}^{\beta}\left(K_{1,2}\right)$, что $f=f_{1}+f_{2}$.

В следующей лемме собраны некоторые простые свойства конусов в $\mathbb{R}^{k}$, необходимые для доказательства теоремы 3.

Лемма 9. Пусть $K_{1}$ и $K_{2}-$ замкнутые конусъь в $\mathbb{R}^{k}$. Тогда:

А. Для любой конической окрестности $W$ конуса $K_{1} \cap K_{2}$ существуют такие конические окрестности $V_{1,2}$ конусов $K_{1,2}$, что $\bar{V}_{1} \cap \bar{V}_{2} \subset W$ (черта обозначает замыкание).

Б. Если $K_{1} \cap K_{2}=\{0\}$, то найдется такое $\theta>0$, что $\delta_{K_{1}}(x) \geqslant \theta|x|$ для любого $x \in K_{2}$.

Доказательство. А. Обозначим через $\mathcal{C}$ множество всех конусов в $\mathbb{R}^{k}$, содержащих начало координат. По условию $K_{1}, K_{2}$ и $W$ принадлежат $\mathcal{C}$. Легко видеть,

\footnotetext{
${ }^{5)}$ Напомним, что прямые суммы конечных семейств пространств DFS и замкнутые подпространства пространств DFS есть снова пространства DFS (см. [12]).
} 
что отображение $U \rightarrow \operatorname{Pr} U$ является биекцией между $\mathcal{C}$ и множеством всех подмножеств сферы $\mathbb{S}_{k-1}=\left(\mathbb{R}^{k} \backslash\{0\}\right) / \mathbb{R}_{+}$. Обозначим через $Q$ обратное к нему отображение. Нетрудно проверить, что оба отображения $\operatorname{Pr}$ и $Q$ сохраняют замыкания, объединения и пересечения. Поэтому множества $\operatorname{Pr} K_{1,2}$ замкнуты, и справедливо включение $\operatorname{Pr} K_{1} \cap \operatorname{Pr} K_{2} \subset \operatorname{Pr} W$. Поскольку сфера $\mathbb{S}_{k-1}$ компактна, существуют такие открытые окрестности $O_{1,2}$ множеств $\operatorname{Pr} K_{1,2}$ в $\mathbb{S}_{k-1}$, что $\bar{O}_{1} \cap \bar{O}_{2} \subset \operatorname{Pr} W$. Положим $V_{1,2}=Q\left(O_{1,2}\right)$. Тогда $\bar{V}_{1} \cap \bar{V}_{2}=Q\left(\bar{O}_{1} \cap \bar{O}_{2}\right) \subset Q(\operatorname{Pr} W)=W$.

Б. Пусть $K_{2} \neq\{0\}$ (если $K_{2}=\{0\}$, то утверждение верно для любого $\theta>0$ ). Положим $F=\left\{x \in \mathbb{R}^{k}: x \in K_{2}\right.$ и $\left.|x|=1\right\}$ и $\theta=\inf _{x \in F} \delta_{K_{1}}(x)$. Так как множество $F$ компактно и $F \cap K_{1}=\varnothing$, то $\theta>0$. Остается заметить, что $\delta_{K_{1}}(x)=|x| \delta_{K_{1}}(x /|x|) \geqslant$ $\theta|x|$ для любого ненулевого $x \in K_{2}$.

Лемма 10. Пусть $A, B>0$, а $U_{1}, U_{2}$ и $U$ - такие конусы в $\mathbb{R}^{k}$, что $\bar{U}_{1} \cap \bar{U}_{2}=$ $\{0\}$. Предположим, что пространство $\mathcal{E}_{\alpha}^{\beta}\left(\mathbb{R}^{k}\right)$ нетривиально. Тогда для любого $f \in H_{\alpha, A}^{\beta, B}(U)$ найдутся такие $A^{\prime}, B^{\prime}>0 u f_{1,2} \in H_{\alpha, A^{\prime}}^{\beta, B^{\prime}}\left(U \cup U_{1,2}\right)$, чmo $f=f_{1}+f_{2}$.

Доказательство. Существуют такие конические окрестности $V_{1,2}$ конусов $\bar{U}_{1,2}$ и такие измеримые конусы $W_{1,2}$, что

$$
W_{1} \cup W_{2}=\mathbb{R}^{k}, \quad W_{1} \cap W_{2}=\{0\}, \quad \bar{V}_{\nu} \cap \bar{W}_{\nu}=\{0\}, \quad \nu=1,2 .
$$

В самом деле, применяя утверждение А леммы 9 к замкнутым конусам $\bar{U}_{1}$ и $\bar{U}_{2}{ }^{6}$, найдем такие конические окрестности $V_{1,2}$ конусов $\bar{U}_{1,2}$, что $\bar{V}_{1} \cap \bar{V}_{2}=\{0\}$. Снова применяя утверждение А леммы $9 \mathrm{k} \bar{V}_{1,2}$, заключаем, что существует такая коническая окрестность $W_{2}$ конуса $\bar{V}_{1}$, что $\bar{V}_{2} \cap \bar{W}_{2}=\{0\}$. Положим $W_{1}=\left(\mathbb{R}^{k} \backslash W_{2}\right) \cup\{0\}$. Тогда первые два соотношения в (28) очевидным образом выполнены, и $\bar{V}_{1} \cap \bar{W}_{1}=$ $\bar{V}_{1} \cap W_{1}=\{0\}$, поскольку конус $W_{1}$ замкнут.

Пусть $g_{0}$ - такая неотрицательная гладкая функция на $\mathbb{R}^{k}$, что $g_{0}(x)=0$ при $|x| \geqslant 1$ и

$$
\int_{\mathbb{R}^{k}} g_{0}(x) d x=1 .
$$

Определим гладкие функции $g_{1}$ и $g_{2}$ на $\mathbb{C}^{k}$ посредством соотношений

$$
g_{\nu}(x+i y)=\int_{W_{\nu}} g_{0}(x-\xi) d \xi, \quad x, y \in \mathbb{R}^{k}, \quad \nu=1,2 .
$$

В силу (28) имеем $g_{1}+g_{2}=1$. Применяя утверждение Б леммы 9 к замкнутым конусам $\bar{U}_{\nu}$ и $\left(\mathbb{R}^{k} \backslash V_{\nu}\right) \cup\{0\}$, заключаем, что существует такое $\theta \in(0,1)$, что $\delta_{U_{\nu}}(x) \geqslant$ $\theta|x|$ при $x \notin V_{\nu}, \nu=1,2$. Так как $\delta_{U}(x) \leqslant|x|$ для любого $x \in \mathbb{R}^{k}$, то

$$
\delta_{U}(\theta x) \leqslant \min \left(\delta_{U}(x), \theta|x|\right) \leqslant \min \left(\delta_{U}(x), \delta_{U_{\nu}}(x)\right)=\delta_{U \cup U_{\nu}}(x), \quad x \notin V_{\nu} .
$$

Пусть $\widetilde{W}_{\nu}=\left\{x \in \mathbb{R}^{k}: \delta_{W_{\nu}}(x) \leqslant 1\right\}, \nu=1,2$. Из (28) и утверждения Б леммы 9 вытекает, что найдется такое $\theta^{\prime}>0$, что $\delta_{W_{\nu}}(x) \geqslant \theta^{\prime}|x|$ при $x \in \bar{V}_{\nu}, \nu=1,2$.

\footnotetext{
6) Заметим, что вырожденный конус $\{0\}$ является конической окрестностью самого себя.
} 
Следовательно, $\delta_{W_{\nu}}(x)>1$ для всех $x \in V_{\nu}$, удовлетворяющих условию $|x| \geqslant 1 / \theta^{\prime}$, т.е. множества $V_{\nu} \cap \widetilde{W}_{\nu}$ ограничены в $\mathbb{R}^{k}$. С учетом (29) отсюда следует, что

$$
\delta_{U}(x) \leqslant \delta_{U \cup U_{\nu}}\left(\frac{x}{\theta}\right)+C, \quad x \in \widetilde{W}_{\nu}, \quad \nu=1,2,
$$

где $C$ - константа. Отсюда находим

$$
\delta_{U}(x) \leqslant \delta_{U \cup U_{1} \cup U_{2}}\left(\frac{x}{\theta}\right)+C, \quad x \in \widetilde{W}_{1} \cap \widetilde{W}_{2} .
$$

Пусть $\tilde{f}_{1,2}=f g_{1,2}$. Так как функция $f$ аналитична, то $\bar{\partial}_{j} \tilde{f}_{1}=f \bar{\partial}_{j} g_{1}, j=1,2, \ldots, k$. По определению $g_{\nu}$ имеем $\operatorname{supp} g_{\nu} \subset \widetilde{W}_{\nu}, \nu=1,2$. Поскольку $g_{1}+g_{2}=1$, отсюда следует, что supp $\bar{\partial}_{j} g_{1} \subset \widetilde{W}_{1} \cap \widetilde{W}_{2}$, и согласно (2), (30) и (31) находим

$$
\left\|\tilde{f}_{\nu}\right\|_{U \cup U_{\nu}, A, \widetilde{B}}^{\prime} \leqslant \widetilde{C}\|f\|_{U, A, B}^{\prime}, \quad\left\|\bar{\partial}_{j} \tilde{f}_{1}\right\|_{U \cup U_{1} \cup U_{2}, A, \widetilde{B}}^{\prime} \leqslant \widetilde{C}\|f\|_{U, A, B}^{\prime}, \quad \nu=1,2,
$$

где $j=1,2, \ldots, k, \widetilde{B}=B / \theta$, а $\widetilde{C}$ - положительная константа. Как было показано при доказательстве утверждения 1 теоремы 1 , нетривиальность пространства $\mathcal{E}_{\alpha}^{\beta}\left(\mathbb{R}^{k}\right)$ влечет существование целой функции $\varphi$ на $\mathbb{C}$, удовлетворяющей условию (5). В силу леммы 2 и следствия 2 найдутся такие $A^{\prime} \geqslant A, B^{\prime} \geqslant \widetilde{B}$ и плюрисубгармоническая функция $\rho$, что

$$
\rho_{U \cup U_{1} \cup U_{2}, A, \widetilde{B}}(z)-H \leqslant \rho(z) \leqslant \rho_{U \cup U_{1} \cup U_{2}, A^{\prime}, B^{\prime}}(z)-\ln \left(1+|z|^{2}\right), \quad z \in \mathbb{C}^{k},
$$

где $H$ - константа. Из (32) и (33) следует, что

$$
\int\left|\bar{\partial}_{j} \tilde{f}_{1}(z)\right|^{2} e^{-2 \rho(z)} d \lambda(z)<\infty .
$$

В силу леммы 7 у неоднородных уравнений Коши-Римана $\bar{\partial}_{j} \psi=\bar{\partial}_{j} \tilde{f}_{1}$ есть такое локально квадратично-интегрируемое решение, что

$$
\int|\psi(z)|^{2} e^{-2 \rho(z)}\left(1+|z|^{2}\right)^{-2} d \lambda(z)<\infty .
$$

Имеем $\bar{\partial}_{j}\left(\tilde{f}_{1}-\psi\right)=\bar{\partial}_{j}\left(\tilde{f}_{2}+\psi\right)=0$, и, следовательно, существуют целые аналитические функции $f_{1}$ и $f_{2}$, совпадающие почти везде с $\tilde{f}_{1}-\psi$ и $\tilde{f}_{2}+\psi$, соответственно. Из правого неравенства в (33) и условия (34) вытекает, что $\|\psi\|_{U \cup U_{1} \cup U_{2}, A^{\prime}, B^{\prime}}^{\prime}<\infty$. Ввиду (32) отсюда следует, что $f_{\nu} \in H_{\alpha, A^{\prime}}^{\beta, B^{\prime}}\left(U \cup U_{\nu}\right), \nu=1,2$. Для завершения доказательства остается заметить, что $f=f_{1}+f_{2}$, поскольку непрерывные функции, совпадающие почти везде, равны.

ДоКАЗАТЕЛЬСТво теОРемы 3. В силу леммы 4 существуют такие $A, B>0$ и коническая окрестность $W$ конуса $K_{1} \cap K_{2}$, что $f \in H_{\alpha, A}^{\beta, B}(W)$. Согласно утверждению А леммы 9 найдутся такие конические окрестности $V_{1,2}$ конусов $K_{1,2}$, что $\bar{V}_{1} \cap \bar{V}_{2} \subset W$. Так как $W$ имеет открытую проекцию, то конус $V=\left(\mathbb{R}^{k} \backslash W\right) \cup\{0\}$ замкнут. Применяя лемму 10 к замкнутым конусам $U_{1,2}=\bar{V}_{1,2} \cap V$ (очевидно, $\left.U_{1} \cap U_{2}=\{0\}\right)$, найдем такие $A^{\prime}, B^{\prime}>0$ и $f_{1,2} \in H_{\alpha, A^{\prime}}^{\beta, B^{\prime}}\left(W \cup U_{1,2}\right)$, что $f=f_{1}+f_{2}$. Поскольку $W \cup U_{1,2} \supset V_{1,2}$, из леммы 4 следует, что $f_{1,2} \in \mathcal{E}_{\alpha}^{\beta}\left(K_{1,2}\right)$. Это завершает доказательство теоремы 3 и утверждения 3 теоремы 1. 
Благодарности. Данная работа была поддержана РФФИ (грант № 05-01-01049), INTAS (грант № 03-51-6346), Программой поддержки ведущих научных школ (грант № НШ-4401.2006.2) и грантом МК-1315.2006.1 Президента РФ.

\section{Список литературы}

[1] Л. Хермандер, Анализ линейных дифференциальных операторов с частными производными. Т. 1. Теория распределений и анализ Фуръе, Мир, М., 1986.

[2] A.Z. Capri, R. Ferrari, J. Math. Phys., 25 (1984), 141.

[3] Р. Ф. Стритер, А. С. Вайтман, РCТ, спин и статистика и все такое, Наука, М., 1966.

[4] E. Brüning, S. Nagamachi, J. Math. Phys., 30 (1989), 2340.

[5] U. Moschella, F. Strocchi, Lett. Math. Phys., 24 (1992), 103.

[6] M. A. Soloviev, Lett. Math. Phys., 33 (1995), 49.

[7] M. A. Soloviev, Commun. Math. Phys., 184 (1997), 579.

[8] И. М. Гельфанд, Г. Е. Шилов, Обобщенные функиии. Вып. 2. Пространства основных и обобщенных функиий, Физматгиз, М., 1958.

[9] А. Г. Смирнов, М. А. Соловьев, ТМФ, 125:1 (2000), 57.

[10] И. М. Гельфанд, Г. Е. Шилов, Обобщенные функиии. Вып. 3. Некоторые вопросы теории дифференциальных уравнений, Физматгиз, М., 1958.

[11] A.G. Smirnov, On topological tensor products of functional Fréchet and DF spaces, math.FA/0512611.

[12] H. Komatsu, J. Math. Soc. Japan, 19 (1967), 366.

[13] Х. Шефер, Топологические векторные пространства, Мир, М., 1971.

[14] В. С. Владимиров, Методы теории функиий многих комплексных переменных, Наука, M., 1964.

[15] L. Hörmander, Notions of Convexity, Progr. Math., 127, Birkhäuser, Boston, 1994.

Поступила в редакцию 3.10 .2006 